\title{
U so de metotrexato en pacientes con lupus eritematoso sistémico
}

\author{
Yunchoy Sánchez Ha, Aurelio Carvallo V. \\ Methotrexate use in patients \\ with systemic lupus erythematosus
}

\begin{abstract}
Background: In the past ten years, methotrexate (MTX) has appeared as an alternative for the treatment of systemic lupus erythematosus (SLE). Aim: To evaluate the use of MTX and the results of treatment in a group of patients with SLE. Patients and Methods: Retrospective review of 426 files of patients with SLE. Of these, all patients treated with MTX were selected. A review protocol was designed, stating sex, age, time of disease evolution, previous treatment, MTX prescription, doses, effectiveness and side effects. Results: Seven patients were selected. All were women, with an age range of 26 to 62 years old and with 5 to 34 years of disease evolution. Previous treatment with non steroidal anti-inflammatory drugs, steroids, hydroxychloroquine and azathioprine ranged from 6 to 631 months (average $=147.3$ ). Persistent joint and/or skin manifestations were the main cause for the use of MTX. The dose ranged from 7.5 to $15 \mathrm{mg}$ and the treatment lapse from 6 to 106 months. It was effective in all patients, allowing a reduction in prednisone doses. Two patients experienced a rise in serum transaminases and one had gastric intolerance, that required treatment discontinuation. Conclusions: MTX is rarely used in SLE. Its main prescription and effectiveness is in joint and skin manifestations, allowing to decrease steroid doses. However, side effects are frequent (Rev Méd Chile 2004; 132: 195-201).
\end{abstract}

(Key Words: Lupus erythematosus, systemic; Methotrexate; Prednisone)

Recibido el 8 de julio, 2003. Aceptado en versión corregida el 7 de octubre, 2003. Departamento de Reumatología, Hospital San Juan de Dios. Santiago de Chile. aBecaria de Reumatología

$\mathrm{E}^{1}$ lupus eritematoso sistémico (LES) es una enfermedad inflamatoria, multisistémica, de etiología desconocida, que se caracteriza por una amplia respuesta autoinmune que induce múltiples injurias tisulares. Por su etiología no precisada, el tratamiento se ha orientado a controlar sus

Correspondencia a: Dr. Aurelio Carvallo V. Departamento de Reumatología, Hospital San Juan de Dios. Chacabuco 419, Santiago de Chile. Fax: 6816430. manifestaciones clínicas e influir sobre los diferentes mecanismos patogénicos, a través de los cuales el o los agentes etiológicos ocasionan el daño. El tratamiento clásico del LES depende de sus características clínicas, por lo que éste varía desde el empleo de antiinflamatorios no esteroidales (AINES) hasta corticoesteroides, antimalári$\cos$ e inmunosupresores; estos últimos, como azatioprina, ciclofosfamida y ciclosporina A, se caracterizan porque, además de su efectividad, presentan un significativo grado de toxicidad y riesgo de oncogenicidad tardía ${ }^{1-3}$. 
El metotrexato (MTX), conocido inmunosupresor de primera elección en tratamientos de artritis reumatoidea $(\mathrm{AR})$, artritis crónica juvenil y artritis psoriática, últimamente es utilizado, además, en polimiositis y síndrome de Reiter ${ }^{2,3}$. Miescher y col, en 1965, comunican por primera vez el uso de MTX en pacientes con LES; sin embargo, de acuerdo a la literatura, sólo recientemente se le ha asignado a este fármaco un rol más preponderante en esta patología $2-7$. Se ha demostrado que sobre $80 \%$ de los lúpicos pueden desarrollar artritis, con secuelas deformantes en 5 a $10 \%$ de ellos $^{2-8}$ y sería preferentemente sobre estas manifestaciones y las cutáneas en las que tendría su acción más eficaz el fármaco. El mecanismo de acción del MTX es como antifólico, interfiriendo las síntesis de ADN y ARN al inhibir la enzima folatorreductasa; también se ha planteado que tiene un efecto antiinflamatorio a través de la inhibición de la quimiotaxis de los ventrófilos y la formación de leucotrienos ${ }^{5,9-10}$. Experiencias con ratones lúpicos tratados con MTX evidencian disminución de la interleukina (IL-1) y el factor de necrosis tumoral alfa $(\mathrm{TNF} \alpha)^{4,11}$. Es, además, una alternativa válida frente a drogas más tóxicas y un importante ahorrador del uso de corticoesteroides $1,5,9,10,12,13-15$.

La literatura de la experiencia internacional del uso de MTX en LES, a nivel clínico, hasta el momento ha sido escasa; sus comunicaciones reúnen entre 7 y 24 pacientes por serie, sumando un total de sólo 207 pacientes hasta enero de 2001. No hay hasta el presente, publicaciones a nivel nacional.

Los antecedentes señalados hacen interesante, por lo tanto, comunicar nuestra experiencia retrospectiva, y aun reducida, de 7 pacientes tratados con MTX, de un universo de 426 lúpicos, controlados en el Departamento de Reumatología del Hospital San Juan de Dios.

\section{Pacientes y MÉtodos}

Se realizó una revisión retrospectiva de 426 fichas clínicas de pacientes lúpicos, controlados en el Departamento de Reumatología del Hospital San Juan de Dios de Santiago. De éstas, se seleccionaron siete fichas correspondientes a todos los pacientes lúpicos tratados con MTX, durante pe- ríodos variables entre marzo de 1993 y marzo de 2002. El diagnóstico de LES se efectuó de acuerdo a los criterios del American College of Rheumatology (ACR, 1982) ${ }^{2}$. Se descartaron pacientes que tuvieron sobreposición con otra afección autoinmune del tejido conectivo.

Se diseñó un protocolo al que se transcribieron los antecedentes de edad, sexo, tiempo de evolución de la enfermedad, criterios diagnósticos y tratamientos previos; se consignó la indicación de MTX, su dosis de inicio, duración, respuesta clínica y parámetros de laboratorio, así como causas de discontinuidad de tratamiento y reacciones adversas a éste.

Los antecedentes de cada ficha, consignados en el protocolo, se ordenaron según cada parámetro en estudio y se analizaron sus resultados.

\section{RESULTADOS}

Sexo, edad y tiempo de evolución de la enfermedad. Todos los pacientes seleccionados correspondieron al sexo femenino; sus edades fluctuaron entre 26 y 62 años $(\bar{x}=42,9)$ y la evolución clínica de LES varió de 5 a 34 años $(\bar{x}=14,4)$.

Características clínicas y criterios de clasificación de LES. En relación a los criterios de clasificación de LES, estos pacientes reunieron entre 5 y 10 de ellos (Tabla 1). Todos los pacientes presentaron en algún momento de su evolución eritema malar, artritis, compromiso hematológico y presencia de anticuerpos antinucleares (ANA); en $6 / 7$, fotosensibilidad, en $6 / 7$, presencia de uno o más marcadores inmunológicos (anti-ADN, anti-Sm, antifosfolípidos); en 5/7, serositis; en 4/7, compromiso neurológico caracterizado por síndrome depresivo orgánico, síndrome de hipertensión endocraneana subaguda, meningitis aséptica y polineuritis de extremidades inferiores; 2/7, compromiso renal cuyas biopsias correspondieron a la clase II y V (OMS) inactivos desde el punto de vista renal y en $1 / 7$, eritema discoide o úlcera oral, además uno de los pacientes presentó compromiso pleuropulmonar y se complicó con empiema y neumotórax, necesitando toracotomía. Otros elementos clínicos encontrados fueron vasculitis en extremidades inferiores y superiores, localizándo- 
Tabla 1. C riterios de clasificación de LES (ACR 1982) de los pacientes tratados con M T X

\begin{tabular}{|llllllllc|}
\hline Manifestaciones/Pac. & 1 & 2 & 3 & 4 & 5 & 6 & 7 & Total \\
\hline Eritema malar & + & + & + & + & + & + & + & 7 \\
Eritema discoide & - & - & - & - & - & + & - & 1 \\
Fotosensibilidad & + & + & + & - & + & + & + & 6 \\
Ulceras orales & - & - & + & - & - & - & - & 1 \\
Artritis no erosiva & + & + & + & + & + & + & + & 7 \\
Serositis* & - & + & + & + & - & + & + & 5 \\
Compromiso renal** & - & - & + & + & - & - & - & 2 \\
C. Neurológico*** & + & - & + & + & - & - & + & 4 \\
C. Hematológico*1*4* & + & + & + & + & + & + & + & 7 \\
Alt. Inmunológico**4*4* & + & + & + & + & - & + & + & 6 \\
ANA (+) & + & + & + & + & + & + & + & 7 \\
Total (+) & 7 & 7 & 10 & 8 & 5 & 8 & 8 & \\
\hline
\end{tabular}

*Pleuritis, pericarditis. **Proteinuria mayor de $0,5 \mathrm{~g} / 24 \mathrm{~h}$, cilindros celulares. ${ }^{* *}$ Convulsiones psicosis. ****Anemia hemolítica con reticulocitosis, leucopenia menor de $4.000 / \mathrm{mm}^{3}$, linfopenia menor de $1.500 / \mathrm{mm}^{3}$, trombocitopenia menor de $100.000 / \mathrm{mm}^{3}$ en ausencia de drogas sospechosas. **akélulas LE, Ac antiADN nativo, Ac anti Sm, Ac antifosfolípidos.

se estas últimas en dedos, dorso de manos y hombros; también a nivel facial, en párpados; cuello y cuero cabelludo. El compromiso del estado general se caracterizó por fiebre de hasta $40^{\circ}$, baja de peso, astenia y adinamia.

Marcadores inmunológicos. Los principales marcadores inmunológicos que presentaron los pacientes lúpicos, previo al uso de MTX, se presentan en la Tabla 2. Todos los pacientes presentaron $\mathrm{ANA}(+), 5 / 7$ aADN(+) y todos tenían complemento bajo previo al uso de MTX.

Tratamiento previo. Los tratamientos previos al uso de MTX en estos pacientes se resumen en la Tabla 3. Se puede observar que todos los pacientes habían usado corticoesteriodes vía oral o parenteral. Las dosis fluctuaron entre $0,5-1 \mathrm{mg} / \mathrm{k}$ de peso diario de prednisona o equivalente, a excepción de un paciente (№ 5) que había

Tabla 2. M arcadores inmunológicos en pacientes lúpicos previo al uso de metotrexato

\begin{tabular}{|llllllll|}
\hline Laboratorio/paciente & 1 & 2 & 3 & 4 & 5 & 6 & 7 \\
ANA & $(+)$ & $(+)$ & $(+)$ & $(+)$ & $(+)$ & $(+)$ & $(+)$ \\
& $15,5^{*}$ & $1 / 100^{* *}$ & $1 / 160$ & $14^{*}$ & $1 / 160$ & 5.120 & $1 / 80$ \\
Anti DNA & $(+)$ & $(+)$ & $(+)$ & $(+)$ & $(-)$ & $(+)$ & $(-)$ \\
C3 C4 & $81,5^{*}$ & $137^{*}$ & débil*** & $280^{*}$ & & $76,1^{*}$ & \\
& $\downarrow \downarrow$ & $\downarrow \downarrow$ & $\downarrow \downarrow$ & $\downarrow \downarrow$ & $\downarrow$ & $\downarrow \downarrow$ & $\downarrow \downarrow$ \\
& $55 / 9$ & $60 / 15$ & $35 / 7,2$ & $56 / 6,4$ & $(\mathrm{CH} 50)$ & $66 / 12$ & $49 / 6$ \\
\hline
\end{tabular}

*ANA (ELSA normal $\leq 1,0$ ). dsDNA: negativo 0 -30; dudoso 31-75; positivo $\geq 76$. *Inmunofluorescencia. ***Chritidia Lucilae C3: Normal 70-140/C4: Normal 10-84. 
Tabla 3. Tratamiento previo y tiempo de duración del LES

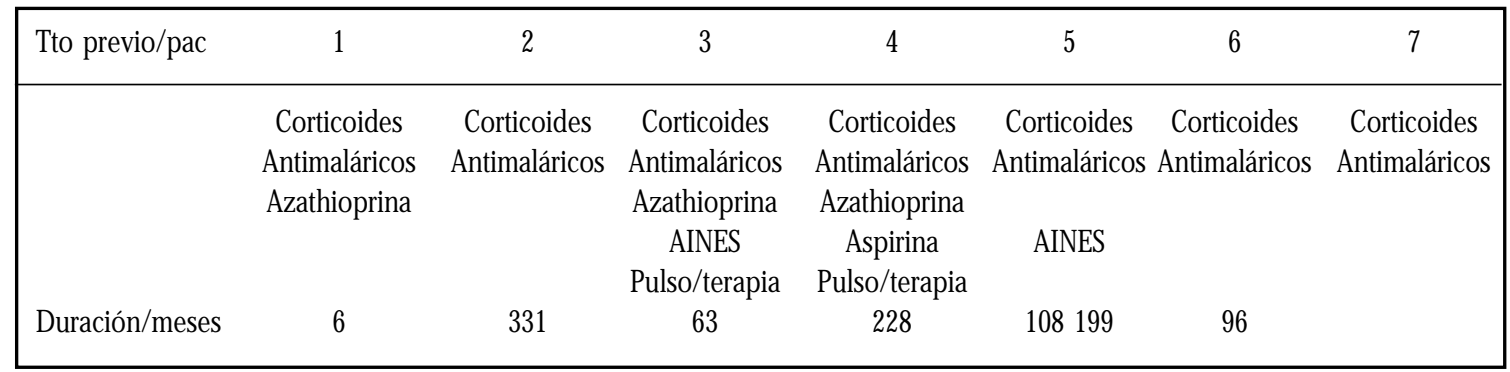

recibido una dosis más baja (20 mg de prednisona y otras 2 (№ 3 y 4) que recibieron además pulsoterapia (compromiso renal). Todas las pacientes habían recibido antimaláricos (hidroxicloroquina) en dosis entre 200 y $400 \mathrm{mg}$. En tres de ellas se usó azatioprina y en 3 también AINES, incluyendo un tratamiento con aspirina ${ }^{\circledR}$. El tiempo de tratamiento previo varió entre 6 y 331 meses con un $\bar{x}=147,3$ meses.

Indicaciones para el uso de MTX (se resumen en la Tabla 4). En 5 pacientes la indicación de MTX fue compromiso cutáneo agudo y subagudo, inclu- yendo 3 pacientes con vasculitis severa de manos, hombros, cuello, cuero cabelludo y extremidades inferiores. Cuatro pacientes presentaron manifestaciones articulares incapacitantes, otra de ellas presentó compromiso hematológico secundario al uso de azatioprina (leucopenia y linfopenia) y otra tenía, además, síntomas constitucionales. Como se observa en la Tabla 5, existía, en la mayoría, más de una manifestación en el momento de ser establecido el tratamiento.

Dosis y tiempo de uso de MTX (Tabla 4). Las dosis de inicio fueron entre 7,5 y $10 \mathrm{mg}$ semanales,

Tabla 4. Indicadores para el uso de M T X

\begin{tabular}{|c|c|c|c|c|c|c|c|}
\hline \multirow{4}{*}{$\begin{array}{l}\text { Variable/paciente } \\
\text { indicación MTX }\end{array}$} & 1 & 2 & 3 & 4 & 5 & 6 & 7 \\
\hline & $\begin{array}{l}\text { Hematológico } \\
\text { Articular }\end{array}$ & $\begin{array}{l}\text { S contitucional } \\
\text { Articular }\end{array}$ & & & \multirow[t]{3}{*}{ Articular } & & \multirow[t]{3}{*}{ Articular } \\
\hline & Cutáneo & Cutáneo & Cutáneo & Cutáneo & & Cutáneo & \\
\hline & & & Vasculitis & Vasculitis & & Vasculitis & \\
\hline \multicolumn{8}{|c|}{ Dosis MTX/mg/sem } \\
\hline & 7,5 & 7,5 & $10-15$ & 10-15 & $7,5-5$ & $7,5-10$ & 7,5 \\
\hline \multicolumn{8}{|c|}{ Tiempo de uso/meses } \\
\hline & 19 & 6 & 106 & 22 & 83 & 31 & 24 \\
\hline \multicolumn{8}{|l|}{ RAM } \\
\hline & & $\uparrow$ GOT-GPT & & & $\uparrow$ GOT-GPT & $\uparrow$ GOT-GPT & GI \\
\hline
\end{tabular}


aumentando éstas hasta $15 \mathrm{mg}$ ( 2 pacientes), de acuerdo a la evolución. Durante este período todos mantuvieron tratamiento con corticoesteroides.

El tiempo de uso del medicamento varió entre 6 y 106 meses.

Evolución clínica y continuidad del tratamiento. Todos los pacientes tuvieron una evolución clínica favorable, con inactivación de las causas que llevaron al uso de MTX (artritis, compromiso cutáneo). Los requerimientos de corticoesteroides tuvieron un descenso importante, llegando incluso en uno de los casos (№ 5) a suspenderlo definitivamente (Figura 1). En 4 pacientes se mantuvo el tratamiento con MTX en dosis entre 7,5 y $15 \mathrm{mg}$ semanales, una de ellas suspendió temporalmente el tratamiento por embarazo. En 3 pacientes fue necesario suspender el tratamiento por efectos adversos; en ninguna la suspensión fue por ineficacia.

Efectos adversos. Tres pacientes presentaron alza de transaminasas, siendo 2 de ellas reincidentes, lo que obligó a suspender definitivamente la terapia. Una paciente presentó intolerancia gástrica con suspensión definitiva a los 2 años de tratamiento.
En esta recopilación no se consideraron LES como parte de un síndrome de sobreposición, como señalan otros autores ${ }^{7}$.

\section{DisCUSIÓN}

El MTX, potente antimetabolito que inhibe la síntesis del ácido fólico, ha demostrado ser eficaz en el tratamiento de afecciones reumatológicas como la artritis reumatoidea (AR), la artropatía psoriática y la polidermatomiositis. En los últimos años la investigación ha permitido extender su uso a otras afecciones, como es el caso de la esclerosis sistémica progresiva (ESP) y el LES. La experiencia internacional en relación al LES tratado con MTX se reduce a no más de 15 trabajos clínicos publicados, con un número de pacientes reducido (entre 7 y 24 pacientes) y teniendo como fundamento de su uso preferentemente manifestaciones articulares, cutáneas y ocasionalmente renales.

$\mathrm{Si}$ bien, nuestra experiencia es reducida y retrospectiva, es a su vez, un intento de ordenar y evaluar las causas que llevan a modificar en algunos pacientes las terapias clásicas por otras aún no consensuadas en espera de ver sus resultados clínicos.

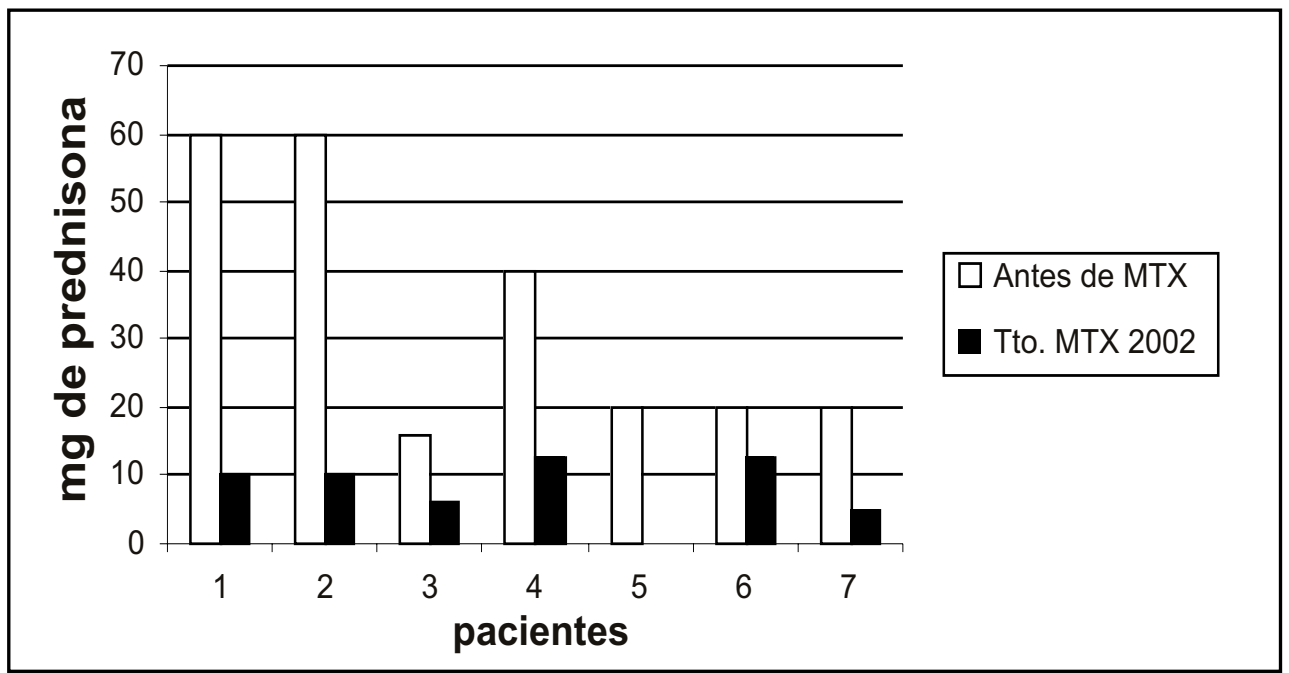

Figura 1. Dosis de prednisona en pacientes LES (antes y después de uso de MTX). 
Así como hay autores que inicialmente proclamaron la eficacia del MTX, usado a dosis alta, en pacientes lúpicos con manifestaciones articulares y cutáneas, como es el trabajo de Miescher y Riethmuller en 1965, que utilizaron $50 \mathrm{mg}$ ev semanales en 10 pacientes ${ }^{16}$, hay otros como Dubois, que señalan manifestaciones tóxicas y ausencia de beneficio en 7 pacientes de los cuales 6 tenían nefropatía ${ }^{17}$. Se puede deducir que la experiencia y conclusiones son diferentes, pero a su vez los grupos no son comparables en lo que a severidad del LES se refiere.

En el último tiempo se han desarrollado experiencias, aún con un número insuficiente de pacientes, que plantean al MTX como una alternativa promisoria en manifestaciones lúpicas especialmente articulares y cutáneas.

En nuestra revisión y como es ya clásico, las 7 pacientes fueron de sexo femenino. Llama la atención el prolongado tiempo de evolución de la enfermedad (5 a 34 años) que llevaban cuando se decidió cambiar la terapia con MTX. La explicación está en primer lugar, en la falta de respuesta satisfactoria al tratamiento previo, en lo que se refiere a manifestaciones articulares y cutáneas, y muy relacionado con esto, en la necesidad de usar dosis de corticoesteroides no deseables y desproporcionadas a las manifestaciones predominantes en esa etapa evolutiva como eran las señaladas; en segundo lugar, porque durante un largo tiempo el MTX no se consideró una alternativa válida para ser usada en pacientes lúpicos. Sin embargo, y como ya se señaló, la progresiva experiencia internacional ha permitido orientar su uso precisamente en estas manifestaciones y más aún si no ha habido una respuesta satisfactoria a los corticoides, antimaláricos o inmunosupresores ${ }^{4-8,13-15,17-21}$.

Todas las pacientes de nuestro grupo, excepto una, reunieron 7 o más criterios diagnósticos al inicio o en la evolución de la enfermedad. Esto es, eran enfermas con una afección inicialmente compleja, en las que durante su langa evolución persistieron o reaparecieron manifestaciones preferentemente articulares o cutáneas, que no lograron ser solucionadas en forma satisfactoria con las medidas clásicas.

El rol del MTX en LES con manifestaciones más severas, como renales, neurológicas o hematológicas, no ha sido claramente establecido y son pocos los trabajos orientados en este sentido, incluida una experiencia de Ravelli y cols ${ }^{15}$ en lupus pediátrico en que de 11 casos, 7 tenían nefropatía, no obteniendo una respuesta significativamente favorable.

En todas nuestras pacientes se logró una estabilización inicial de la enfermedad, esto es, la respuesta fue buena, permitiendo incluso disminuir la dosis media de corticoesteroides lo que ha sido señalado en la experiencia internacional $1,5,8,9,11,12,14$.

En relación a los problemas secundarios presentados por 4 de nuestras enfermas, pese a haber logrado beneficio con el tratamiento, 3 de ellas debieron abandonarlo por efectos adversos ya sea hepáticos (alza mantenida de transaminasas en 2 de ellas) o de intolerancia gástrica (1 paciente). Porcentualmente la cifra es alta, pero repetimos, el grupo es pequeño. Si se compara con la experiencia del MTX en la AR, incluida la nuestra21, esta última aparece inferior. La literatura describe problemas adversos frecuentes en el LES tratado con MTX, especialmente hepáticos, gástricos y leucopenia, los que varían entre 27 y $73 \%$, siendo en la mayoría de los casos moderados, sin embargo, en 10 a 33\% de los casos fue necesario discontinuar el tratamiento 1,3-9,11-16. Se ha señalado, como una posibilidad, que algunos de estos pacientes tengan una enfermedad renal subclínica, alterándose de este modo la eliminación del fármaco, lo que contribuiría a los efectos tóxicos ${ }^{7}$. Es necesario por consiguiente, un estricto control de la función renal mientras se usa el MTX. Por otra parte, lo cual no hicimos al menos de forma inicial en algunas de nuestras pacientes, es de utilidad adicionar ácido fólico en 1 ó $2 \mathrm{mg} /$ día, lo que ha demostrado disminuir los efectos secundarios del medicamento $3,5,18$.

En conclusión, esta revisión ha permitido evaluar a un grupo de pacientes lúpicos en quienes se usó MTX como alternativa terapéutica en manifestaciones preferentemente articulares y cutáneas de la enfermedad. Basado en esto, y en las publicaciones internacionales, se puede deducir que el fármaco es útil en estos casos, necesitándose dosis que varían entre 7,5 y $15 \mathrm{mg} /$ semana. El compromiso renal propio del LES y a veces subclínico, podría ser un factor contribuyente a los efectos adversos del MTX en estos pacientes, por lo que la vigilancia de la función renal en estos casos debe ser estricta y periódica. Por el momento no se ha 
demostrado que el MTX sea eficaz en manifestaciones más severas del lupus, como renales, neurológicas o hematológicas.

Creemos que a futuro, con un plan prospectivo y un mayor número de casos se podrían

\section{REFERENCIAS}

1. Rothenberg RJ, Graciano FM, Grandone JT, GolBerg JW, BJarnason L, Finesilver AG. The use of methotrexate in steroid resistant systemic lupus erythematosus. Arthritis Rheum 1988; 31: 612-5.

2. KLPPEL JH. SLE management. En: Klippel JH, Dieppe PA. eds. Rheumatology. St. Louis, London: Mosby 1995.

3. García Carrasco M, Sanmartí R, Cañete J, Alarcón G. Utilización de los principales DMARD. En: Avances en artritis reumatoide. Springer-Verlag Ibérica. Barcelona 2000; 136-51.

4. Gansauge S, Breitbart A, Rinaldi N, Schwarz-Eywill M. Methotrexate in patients with moderate systemic lupus erythematosus (exclusion of renal and central nervous system disease). Ann Rheum Dis 1997; 56: 382-5.

5. Waiz Leblanc BA, Dagenais P, Urowitz MB, GladMAN DD. Methotrexate in systemic lupus erythematosus. J Rheumatol 1994; 21: 836-8.

6. Kimberley W, Micha A. A 2 years, open ended trial of methotrexate in systemic lupus erythematosus. J Rheumatol 1994; 21: 1674-7.

7. Wise C, VuYyuru S, RoberTs N. Methotrexate in nonrenal lupus and undifferentiated connective tissue disease a review of 36 patients. J Rheumatol 1996; 23: 1005-10.

8. Carvallo A, Moreno E. Lupus eristematoso sistémico. En: Aris H, Valenzuela F. (Ed): Reumatología $1^{a}$ ed. Santiago de Chile. Fundación de Investigación y Perfeccionamiento Médico 1995; 165-88.

9. Sato EI. Methotrexate therapy in systemic lupus erythematosus. Lupus 2001; 10: 162-4.

10. Rahman P, Humphrey-Murto S, Gladman DD, UrowITZ MB. Efficacy and tolerability of methotrexate in antimalarical resistant lupus arthritis. J Rheumatol 1998; 25: 243-6.

11. Dayan M, Segal R, Mozes E. Cytokine manipulation by methotrexate treatment in murine experimen- obtener conclusiones más valederas con relación a una terapia que parece ser una alternativa prometedora en determinado grupo de pacientes con LES.

tal systemic lupus erythematosus. J Rheumatol 1997; 24: 1075-81.

12. Hashimoto M, Nokada S, Furuta E, Wada T, Suenaga Y, YASUDA M ET AL. Methotrexate for steroid-resistant systemic lupus erythematosus. Clin Rheumatol 1994; 13: 280-3.

13. Wilke WS, Krall PL, Scheetz RJ, Babiak T, Danao T, MazAnec DJ ET AL. Methotrexate for systemic lupus erythematosus: a retrospect analysis of 17 unselected cases. Clin Exp Rheumatol 1991; 9: 581-7.

14. Bottomley W, Goodfield M. Methotrexate for the treatment of severe mucocutaneous lupus erythematosus. British Journal of Dermatology 1995; 133: 311-4.

15. Ravew A, Baluardini G, Viola S, Vila I, Ruperto N, MARTín. Methotrexate therapy in refractory pediatric onset systemic lupus erythematosus. J Rheumatol 1998; 25: 572-5.

16. Miescher PA, RiethmuLeR D. Diagnosis and treatment of systemic lupus erythematosus. Sem Haematol 1965; 2: 1-8.

17. Dubois EL. Management of discoid and systemic lupus erythematosus. En: Dubois EL (ed): $17^{\circ}$ review of the Current Status of Discoid and Systemic Lupus Erythematosus and their variants. $2^{\text {nd }}$ ed. University of Southern California Press: Los Angeles 1974: 537-612.

18. Carneiro JR, Sato EI. Double blind, randomized, placebo controlled clinical trial of methotrexate in systemic lupus erythematosus. J Rheumatol 1999; 26: 1275-9.

19. Kipen Y, Littlejohn GO, Morand EF. Methotrexate use in systemic lupus erythematosus. Lupus 1997; 6: 385-9.

20. Asherson RA, Schatten S, Hughes GRV. Methotrexate in systemic lupus erythematosus. J Rheumatol 1997; 24: 610-1.

21. Carvalo A, Wolf C, Armas Merino R, Villanueva P, Donoso G, Naquira N et al. Artritis reumatoidea. Eficacia terapéutica del metotrexate y sus efectos hepatotóxicos. Rev Méd Chile 1993; 121: 777-84. 